\title{
2-laser injection-locking configuration for Brillouin fibre sensors
}

\author{
Massimo Facchini, Luc Thévenaz, Jörg Troger, Philippe Robert \\ EPFL, Swiss Federal Institute of Technology, Metrology and Photonics Lab, \\ CH-1015 Lausanne, Switzerland \\ Phone: +41 216934774 Fax: +41 $216932614 \quad$ Email: Luc.Thevenaz@epfl.ch
}

\begin{abstract}
Brillouin sensing has proved this past few years to be an efficient tool for distributed measurements of quantities such as temperature and strain [1]. The main challenge for the proper operation of such a sensor is to generate the suitable pump and probe signals, that must be of high spectral purity and must be spectrally separated by a accurate definite frequency in the $10 \mathrm{GHz}$ range. Many configurations have been proposed to date, using either two distinct spectrally stabilized lasers $[2,3]$ or the sidebands resulting from the modulation of a single laser by an electro-optic modulator [1,4], to mention the most widely used.
\end{abstract}

The two-lasers configuration suffers from stability problem, because the frequency difference must be set with a stability better than $1 \mathrm{MHz}$. This problem was satisfactorily overcome with the sideband technique, but it requires high quality electro-optic modulator with an extinction ratio better than $30 \mathrm{~dB}$. Such modulators are now very difficult to obtain, the telecommunication standard requiring only a $20 \mathrm{~dB}$ extinction ratio.

We propose in this paper a novel method that merges the advantages of these two techniques. It is based on the injection locking of two distinct semiconductor lasers, that makes possible the generation of high purity beat signals in the microwave frequency range. Such a technique can be used either in the pulsed pump-probe technique or the new correlation-based technique [5]. Furthermore novel devices, such as laser modules presenting integrated electro-absorption modulators, can be exploited for achieving efficient and cost effective injection locking schemes.

\section{Principle}

When coherent light from one laser is coupled into the cavity of another laser, the latter may leave its own emission frequency and shift into the frequency of the injected light. The first laser is called the MASTER laser and the light source into which light is coupled is named the SLAVE laser. As the frequency locking of the slave laser is achieved by the injection of an external signal, the technique is referred to as laser injection locking. A suitable light isolation has to be introduced between the two lasers, in order to avoid that the light emitted by the slave laser perturbs the master laser emission.

It has been demonstrated both theoretically and experimentally that an fraction of injected power as small as $10^{-5}$ of the emitted power is sufficient for a convenient locking of a semiconductor laser [6]. With such an amount of injected power the slave leaves its own frequency and locks on the master, provided that the slave free running frequency is within $\pm 1 \mathrm{GHz}$ of the master frequency. In addition the master phase noise is faithfully replicated in the slave emission, so that the beat note shows a great spectral purity. 


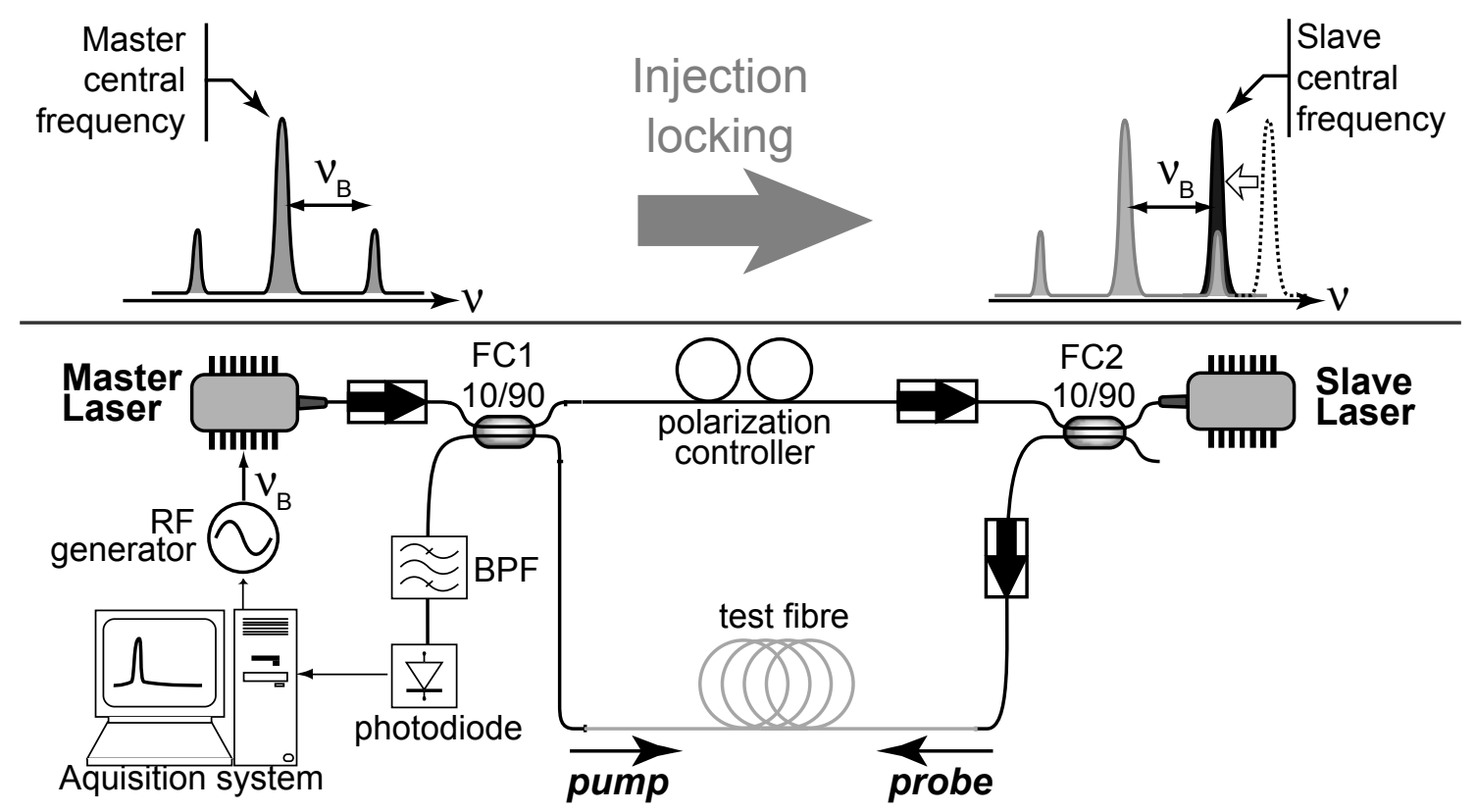

Fig. 1 Experimental setup for locking the unmodulated slave DFB-laser to the sideband of the master laser modulated by a RF signal.

The obtained lightwaves are used as pump and probe signals for generating SBS along an optical fibre.

The two signals separated by a $10 \mathrm{GHz}$ frequency needed for the Brillouin interaction are generated in this case by locking the slave laser on a modulation sideband of the master [7-9]. The modulation depth can be very small as a result of the tiny fraction of injected power needed for achieving proper locking, so that direct modulation of the laser current may be used. The principle of the technique for Brillouin gain measurement is shown in Fig. 1.

It must be pointed out that the locking can be realized by modulating the slave laser and keeping the master unmodulated, as well. In this case the frequency conversion is achieved in the slave cavity, but the principle remains formally identical.

In addition the slave laser can be locked on a higher order sideband, so that the beat note is produced at a multiple of the modulation frequency. This makes possible a lower RF modulation frequency to be used, that may be easier to be generated and fed into the laser. We experimentally achieved successful locking up to the fifth sideband, achieving a 10.825 Ghz optical beat note using a $2.165 \mathrm{GHz}$ RF drive frequency [6].

\section{Results}

A first experiment was carried out to demonstrate the feasibility of this principle for Brillouin gain measurement in optical fibres. The set-up is described in Fig. 1 using a direct modulation of the laser current. The slave was locked in this case on the first sideband, so that the frequency difference between pump and probe is exactly the RF modulation frequency. The strategic placing of isolators must be pointed out and was designed to achieve a pure master-to-slave locking and to avoid any interference through multiple paths.

Two separated fibre path can be observed: the top path is short and is dedicated to direct the master emission into the slave for locking. This path propagates only the master light and contains a polarisation controller to match the master and slave polarisations. The bottom path is the measuring path and both master and slave lights propagate in a counterpropagating configuration to achieve the Brillouin interaction. 


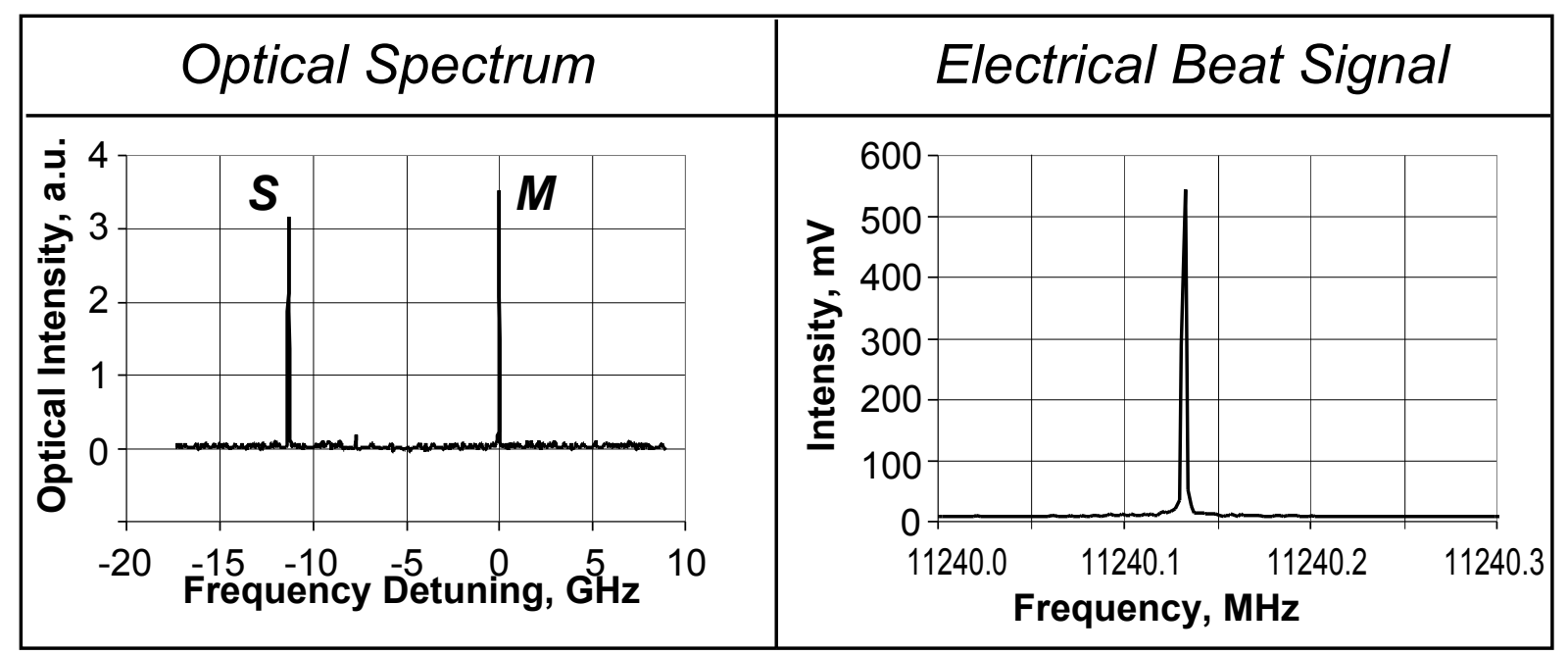

Fig. 2 Spectra of slave and master laser emission under injection locking condition.

The optical spectrum is measured by a Fabry-Perot spectrum analyser (frequency accuracy: $10 \mathrm{MHz}$ or $0.08 \mathrm{pm}$ ). The electrical beat spectrum is observed by means of microwave spectrum analyser.

The optical spectrum of the combined master and slave emissions measured using a FabryPerot analyser is shown in Fig. 2, together with the electrical spectrum of the detected beat note. The excellent spectral purity of the beat signal is clearly demonstrated and the remaining phase noise results in a frequency broadening within the $10 \mathrm{kHz}$ resolution of the spectrum analyser.

Measuring the Brillouin gain spectrum of an optical fibre requires a frequency scanning of the beat note, that is achieved by simply changing the frequency of the RF signal driving the master. The effective locking range around the free running slave frequency is about $2 \mathrm{GHz}$, resulting in comfortable conditions to a proper scan for sensing, the Brillouin linewidth being about $25 \mathrm{MHz}$ and the scanned range for sensing purpose never exceeding $500 \mathrm{MHz}$.

Fig. 3 shows the Brillouin gain spectrum of a $21 \mathrm{~m}$ standard fibre. Brillouin amplification of the probe is clearly observed even at a moderate pump power and over a restricted interaction length. This demonstrates the capability of the technique to potentially measure Brillouin spectra with a meter resolution using a classical pump-probe technique, at the

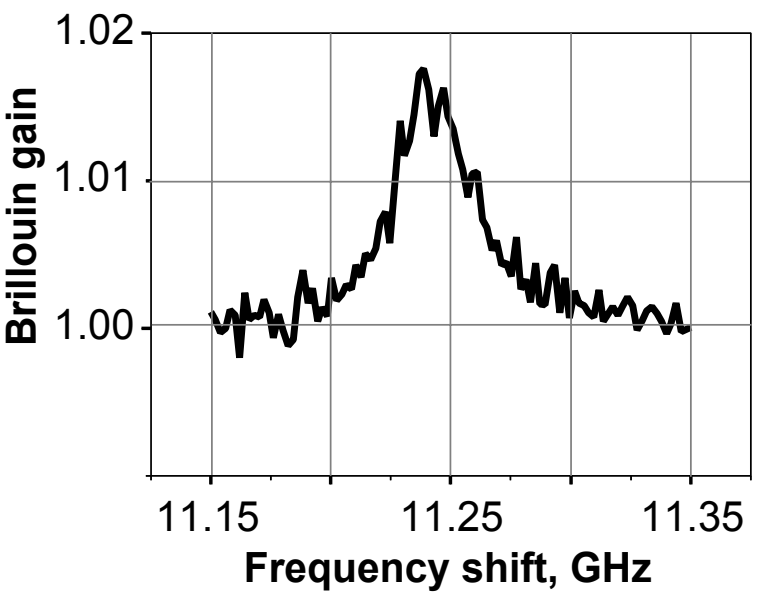

Fig. 3 Brillouin gain curve measured by the phaselocked lasers configuration.

21 metres of standard single-mode fibre are tested using CW pump and probe lightwaves having, respectively, optical powers of $11 \mathrm{~mW}$ and $3 \mathrm{~mW}$. 


\section{Conclusions}

We have demonstrated the capability of an injection-locking configuration for efficient Brillouin spectra measurements for sensing purpose. The experimental set-up is simple and uses devices that are widely available as a result of the telecommunication market demand.

The scheme is flexible and can be easily adapted for distributed measurements, using the very fast locking settling time. Such a configuration is under test and will be reported later. Injection-locking configurations turn out to be also particularly efficient for the novel correlation-based configuration for Brillouin sensing, since the synchronous frequency sweeping of pump and probe signals can be automatically achieved as well.

\section{References}

1. M.Niklès, L.Thévenaz, Ph.Robert, "Brillouin gain spectrum characterization in singlemode optical fibers", J. Lightwave Technol., LT-15, pp. 1842-1851, 1997.

2. X. Bao, J. Dhliwayo, N. Heron, D.J. Webb, D.A. Jackson, "Experimental and theoretical studies on a distributed temperature sensor bases on Brillouin scattering", J. Lightwave Technol., 13, p. 1340, 1995.

3. T.Horiguchi, T.Kurashima, M.Tateda, "A technique to measure distributed strain in optical fibers", Photonics Tech. Lett., 2, p. 352, 1990.

4. M.Niklès, L.Thévenaz, P.Robert, "Simple distributed fiber sensor based on Brillouin gain spectrum a analysis", Optics Lett., 21, pp. 758-760, 1995.

5. K.Hotate, T.Hasegawa, "Measurement of Brillouin Gain Spectrum Distribution along an Optical Fiber with a High Spatial Resolution using a Novel Correlation Technique", Proceedings of13th International Conference on Optical Fiber Sensors, Kyongju (Korea), SPIE Vol. 3746, Washington DC, pp.337-340, 1999.

6. H. J.Troger, "Injection locking in semiconductor lasers", PhD Thesis $N^{\circ}$ 1976, EPFL, Switzerland, 1999.

7. K. Kikuchi, C.-E. Zah, T.-P. Lee, "Amplitude-modulation sideband injection locking characteristics of semiconductor lasers and their application", Journal of Lightwave Technology, 6, pp. 1821-1830, 1988.

8. P. Bouyer, T.L. Gustavson, K.G. Haritos and M.A. Kasevich, "Microwave signal generation with optical injection locking", Optics Letters, 21, pp. 1502-1504, 1996.

9. L. Noel, D. Marcenac and D. Wake, "Optical millimetre-wave generation technique with high efficiency, purity and stability", Electronics Letters, 32, pp. 1997-1998, 1996. 